\title{
Splenic Spontaneous Rupture Associated with Acute Myeloid Leukemia: Report of a Case and Literature Review
}

\section{Fahmi Y*, Elabbasi T, Khaiz D, Bensardi FZ, Hattabi K, Berrada S, Lefriyekh R, Benissa N, Fadil A and Zerouali N}

Service des urgences chirurgicales viscérales, $\mathrm{CHU}$ Ibn Rochd, Casablanca, Morocco

\begin{abstract}
Introduction : Spleen Spontaneous Rupture (SSR) is a rare disease; its diagnosis is difficult. Infectious and hematologic are its most frequent etiologies representing more than the half of cases. The prognosis is depending on quality of care and the nature of the etiology, mortality is high (14\%).
\end{abstract}

Presentation of Case : We report a case of non-traumatic splenic rupture occurring in a woman carrying of acute myeloid leukemia presented with spontaneous rupture of the spleen, and has received an emergency splenectomy.

Discussion : The combination of non-traumatic splenic rupture in acute myeloid leukemia is very rare, and there are some cases reported in the literature regarding this association.

Conclusion : We report this interesting case to emphasize the seriousness of this disease and its various diagnostic and therapeutic modalities.

Keywords: Acute myeloid leukemia; Splenic rupture; Splenectomy

\section{Introduction}

The non-traumatic or Spleen Spontaneous Rupture (SSR) is a very rare and potentially fatal disease entity [1,2]. Delay diagnostic, therapeutic and the severity of the terrain increases mortality of this pathologie it occurs on a spleen disease (tumor) or a healthy rate (during mononucleosis), and it is an indication for emergency splenectomy $[3,4]$. We report the case of a woman followed for acute myéloïd leukemia that was presented with spleen spontaneous rupture to the emergency.

\section{Case Report}

We report a case of a 48 year old patient being treated with good prognosis acute myeloid leukemia (Type 4) who presented with severe abdominal pain without pyrexia. Physical examination showed no evidence of traumatic injury. In clinical examination: the patient was conscious, respiratory rate 30 breaths/min, heart rate 120 beats $/ \mathrm{min}$, blood pressure $09 / 06 \mathrm{~mm}$-hg, temperature was normal, with generalized abdominal tenderness. The patient underwent an abdominal ultrasound that showed an abundance of hemoperitoneum with heterogeneous splenic upper pole. Abdominal scan showed a splenic hematoma with hemoperitoneum of abundance (Figure 1). Hemoglobin was 5.8

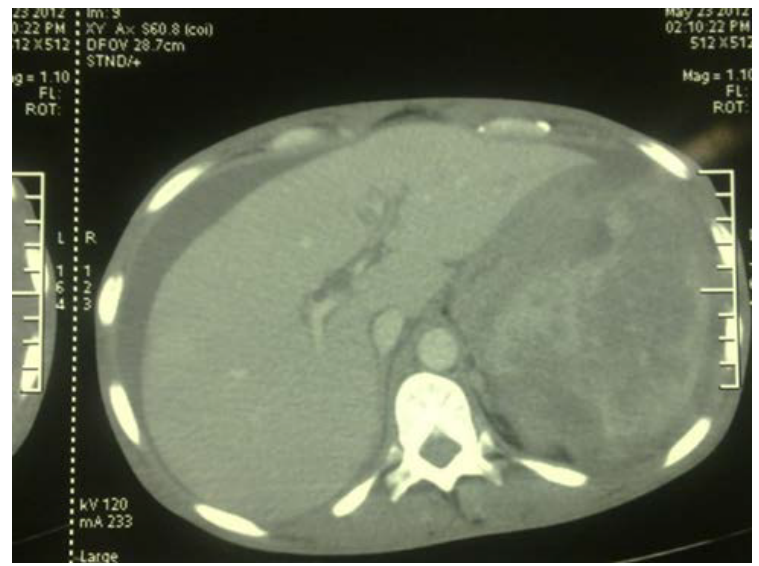

Figure 1: The abdominal CT scan showing an abundance of hemoperitoneum with heterogeneous appearance of the spleen.

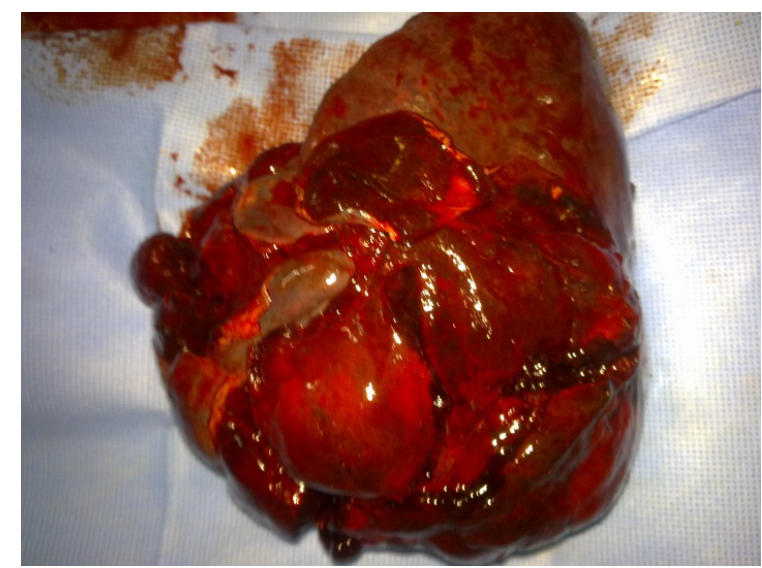

Figure 2: Piece of splenectomywithcapsular rupture of the posterior face.

$\mathrm{g} / \mathrm{dl}, \mathrm{GB}: 1080$ éléments $/ \mathrm{mm}^{3}$, platelets to 8000 cells $/ \mathrm{mm}^{3}$. Given the evolution of the patient to hemodynamic instability, she was transferred to the service of visceral surgical emergencies where she received a splenectomy after transfusion of red blood cells and platelets. Surgical exploration was objectified an hemoperitoneum of abundance, with capsular rupture at the posterior face of the spleen, splenic pedicle was intact (Figures 2 and 3). Post-operative suites were simple, the patient received pneumococcal vaccination/antiheamophilus and oral antibiotics and was transferred to the Division of Hematology for additional care.

${ }^{*}$ Corresponding author: Fahmi Yassine, Service des urgences chirurgicales viscérales (P35), CHU Ibn Rochd, Casablanca, Morocco, Tel: 0664775577; E-mail: fahmi-yassine@hotmail.com

Received December 15, 2013; Accepted January 28, 2014; Published February 05, 2014

Citation: Fahmi Y, Elabbasi T, Khaiz D, Bensardi FZ, Hattabi K, et al. (2014) Splenic Spontaneous Rupture Associated with Acute Myeloïd Leukemia: Report of a Case and Literature Review. Surgery Curr Res 4: 170. doi:10.4172/21611076.1000170

Copyright: ( 2014 Fahmi Y, et al. This is an open-access article distributed under the terms of the Creative Commons Attribution License, which permits unrestricted use, distribution, and reproduction in any medium, provided the original author and source are credited. 


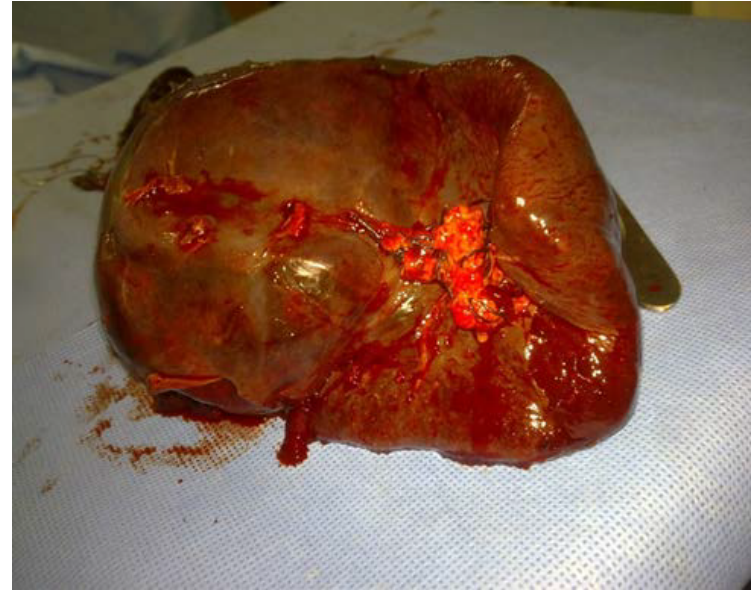

Figure 3: Inner surface of the spleen.

The anapath of the surgical specimen showed signs confirming acute myeloid leukemia type 4

\section{Discussion}

The non-traumatic splenic rupture (SSR) is an emergency diagnostic and therapeutic as noted our observation, it is a very rare cause of spontaneous hemoperitoneum and difficult to diagnose in the absence of an imaging abdominal exam. The first cases of SSR were reported by [1]. The causes of this disease are dominated by infectious and hematologic diseases account for more than half of the cases [1]. Infectious causes (30\%) are mostly represented by mononucleosis infectious and malaria, while hematological causes (27\%) are mainly represented by malignancies peripheral origin , including leukemia and Hodgkin's and non-Hodgkin lymphomas (NHL). Other fewer cases were reported : solid or benign tumors of the spleen (11\%), portal hypertension (10\%), renal insufficiency dialysis stage (3\%). In $5 \%$ of cases, no etiology was found [2]. Hematologic causes are the second etiology of SSR in most cases it's hematological malignancies with a risk of rupture that increase with age [5-7]. Various mechanisms have been proposed : bleeding in infarction sites, in tumor foci and/ or hemostasis disorders. Regarding the SSR in acute myéloïd leukemia incidence is not known and some observations are published. In terms of SSR in hematological malignancies, 136 cases have been reported in literature, acute leukemias represented 34\%. The SSR associated with acute myeloid leukemia affects often adult males without particular explanation [8]. The pathogenesis remains unclear and the mechanisms of leukemic infiltration of caogulopathies and infarct are incriminated.

The SSR may occur in an acute array which corresponds to the spleen immediate rupture associated with abdominal pain syndrome rapidly progressing to collapse and hemorrhagic stock, as was reported the observation of our patient. They can also be revealed by a subacute array corresponding to the spleen rupture in two time and it combines episodes of diffuse abdominal pain with hemodynamic signs such as tachycardia and episodes of hypotension. Data from the physical examination variables : painful splenomegaly in the absence of any trauma, must be systematically suggest the diagnosis. Abdominal ultrasound is the first examination to practice to confirm the diagnosis. When hemodynamic status permits, an abdominal CT scan with injection of contrast can be performed to make an accurate assessment of the lesions, The emergency laparotomy was performed in front of the cases of acute abdominal syndrome with shock allows for hemostasis and confirm the diagnosis. Splenectomy was performed in $90 \%$ of cases, conservative medical treatment without surgery was attempted in less than $7 \%$ of patients [1]. The part of conservative surgery seems very limited in this disease because of its occurrence in the majority of cases in disease or tumoral spleen or infarction seat [1]. In $8 \%$ of cases, patients die before being operated and diagnosis is made at autopsy $[9,10]$. The overall mortality rate reported in the literature is $14 \%$ [11]. After splenectomy regardless of indication, it is conventional to prevent by vaccination against Streptococcus pneumoniae. Infection by Haemophilus influenzae and Neisseria meningitidis are also possible. Some etiologies of SSR especially tumor, probably exposed to an increased risk of postsplenectomy infection [12] risk. It is therefore justified to perform an additional vaccination against these germs, in patients considered immunocompromised and meanwhile these vaccinations be fully effective, prescribe a prophylaxis oral antibiotic (penicillin) that effectiveness is however imperfect due to the increased resistance of causative germs to penicillin [9].

\section{Conclusion}

SSR entities are rare, with high mortality and difficult to diagnose. The overall mortality rate is about $14 \%$ and appears to be primarily related to diagnostic and/or severity of the pathology behind. Infectious etiologies, dominated by MNI and hematological etiologies dominated by hematological malignancies that presented more than half of cases in SSR. The diagnosis must be evoked in front of hemorrhagic shock associated with abdominal pain in the absence of trauma injury. However, a rupture in two times is possible. The diagnosis is based on ultrasound or abdominal CT scan. The treatment is splenectomy, conservative treatment can be offered to selected patients with MNI.

\section{Conflits of Interest}

The authors declare that there is no conflict of interest

\section{Ethical Approval}

Written informed consent has been obtained by the patient.

\section{Author Contributions}

Fahmi Yassine and El Abbassi tawfik contributed to the writing of this paper F.Z. Bensardi, K. Hattabi, S. Berrada, R. Lefriyekh, N. Benissa., A. Fadil, N. Zerouali performed the operation. D. Khaiz supervising the article.

\section{References}

1. Gedik E, Girgin S, Aldemir M, Keles C, Tuncer MC, et al. (2008) Non-traumatic splenic rupture: report of seven cases and review of the literature. World J Gastroenterol 14: 6711-6716.

2. Kianmanesh R, Aguirre HI, Enjaume F, Valverde A, Brugière O, et al. (2003) [Spontaneous splenic rupture: report of three new cases and review of the literature]. Ann Chir 128: 303-309.

3. Farley DR, Zietlow SP, Bannon MP, Farnell MB (1992) Spontaneous rupture of the spleen due to infectious mononucleosis. Mayo Clin Proc 67: 846-853.

4. Lam CM, Yuen ST, Yuen WK (1998) Hemoperitoneum caused by spontaneous rupture of a true splenic cyst. Hepatogastroenterology 45: 1884-1886.

5. Bell JS, Mason JM (1980) Sudden death due to spontaneous rupture of the spleen from infectious mononucleosis. J Forensic Sci 25: 20-24.

6. Karakousis CP, Elias EG (1974) Spontaneous (pathologic) rupture of spleen in malignancies. Surgery 76: 674-677.

7. Haj M, Zaina A, Wiess M, Cohen I, Joseph M, et al. (1999) Pathologicspontaneous-rupture of the spleen as a presenting sign of splenic T-cell lymphoma--case report with review. Hepatogastroenterology 46: 193-195.

8. Giagounidis AA, Burk M, Meckenstock G, Koch AJ, Schneider W (1996) Pathologic rupture of the spleen in hematologic malignancies: two additional cases. Ann Hematol 73: 297-302. 
Citation: Fahmi Y, Elabbasi T, Khaiz D, Bensardi FZ, Hattabi K, et al. (2014) Splenic Spontaneous Rupture Associated with Acute Myeloïd Leukemia: Report of a Case and Literature Review. Surgery Curr Res 4: 170. doi:10.4172/2161-1076.1000170

9. Davidson RN, Wall RA (2001) Prevention and management of infections in patients without a spleen, Clin Microbiol Infect 7: 657-660.

10. Asgari MM, Begos DG (1997) Spontaneous splenic rupture in infectious mononucleosis: a review. Yale J Biol Med 70: 175-182.
11. Conthe P, Cilleros CM, Urbeltz A, Escat J, Gilsanz C (1997) [Spontaneous splenic rupture: surgical or conservative treatment?]. An Med Interna 14: 625-626.

12. Schuler JG, Filtzer H (1995) Spontaneous splenic rupture. The role of nonoperative management. Arch Surg 130: 662-665. 